\title{
A New Wireless Generation Technology for Video Streaming
}

\author{
Sarmad K. Ibrahim $\mathbb{D}^{1}$ and Nasser N. Khamiss ${ }^{2}$ \\ ${ }^{1}$ College of Engineering, Mustansiriyah University, Baghdad, Iraq \\ ${ }^{2}$ College of Information Engineering, Al-Nahrain University, Baghdad, Iraq \\ Correspondence should be addressed to Sarmad K. Ibrahim; eng_sarmadnet@uomustansiriyah.edu.iq
}

Received 31 January 2019; Revised 6 March 2019; Accepted 18 March 2019; Published 21 April 2019

Academic Editor: Youyun Xu

Copyright (c) 2019 Sarmad K. Ibrahim and Nasser N. Khamiss. This is an open access article distributed under the Creative Commons Attribution License, which permits unrestricted use, distribution, and reproduction in any medium, provided the original work is properly cited.

\begin{abstract}
With the exponential rise in the volumes of video traffic in cellular networks, there is an urgent need for improving the quality of video delivery. This research proposes a mobile generation model based on the updated technologies of the fourth- and fifthgeneration mobile systems, which is called Proposed Generation (Pro-G). This model uses wider bandwidth and advanced adaptive modulation and coding. It also incorporates the method of the adaptive video streaming of multiple video data rates by using the transcoding technique, which is called H.265 proposed (H.265 pro). Thus, both methods are tested to provide a large number of users of video/data application with more speed and best quality. A comparison with $4 \mathrm{G}$ technology is done to assign the development regarding number of users with data rate. The suggested video coding shows how much the overall system is more reliable over the congested channel than conventional video coding technologies such as high-efficiency video coding (HEVC/H.265) and advanced video coding (AVC/H.264). The results showed that the proposed method of transmitting wireless data is better than the LTE-ADV method. In this method, the rate of data transfer increases by $29 \%$ compared with LTE-ADV, while the bit rate saving was increased to $13 \%$ in the proposed video coding compared with that in the H.265.
\end{abstract}

\section{Introduction}

Currently, there are many mobile and wireless systems such as fourth-generation (4G) systems, which include LongTerm Evolution (LTE), Wi-Fi, and WiMAX. Wireless networks depend on the IP protocol, which means that this protocol in the network layer expresses all data transmission.

$4 \mathrm{G}$ technology is mainly the extension of the thirdgeneration (3G) technology, with additional information measure and services. $4 \mathrm{G}$ technology can provide several advancements to the wireless market, together with downlink information rates [1].

The $5 \mathrm{G}$ systems offer many distinct characteristics which make them stronger and more demanding in the future [2].

The main objective of the $5 \mathrm{G}$ systems is the transfer of data to mobile devices with high quality and efficiency [3]. These services have already been provided, but the provision of high-definition video, multimedia services, and interactive presentation that leads to degradation in service quality may be a significant challenge. Among the services, the video streaming service is preferred, and with the high flow of data and information, the response to users is essential to maintain the quality of service and performance.

There must be improvements to $4 \mathrm{G}$ systems to accommodate the need for video services, which will be $72 \%$ in 2019 [4].

One of the characteristics of video transmission over wireless networks is the availability of large amounts of data for transmission in a short time. The popular video encoding technology is H.264, which provides a useful service yet is impractical in the transmission of high-resolution data.

This paper proposes a new wireless technology, and it has been compared with $4 \mathrm{G}$ system technology. This paper also designs a new multi-video coding, and it has been evaluated with previous video coding methods.

The advanced wireless technology and video coding have been studied in literature as follows: Pandiaraj [5] improved the throughput of coverage area by using relay techniques, compared their power adoption with WiMAX. The architectural evolution of wireless mobile network technology 
from $4 \mathrm{G}$ to $5 \mathrm{G}$ was discussed by Sule and Joshi [1]; Nissel and Rupp [6] proposed a new modulation scheme which combines the advantages of Filter Bank Multicarrier (FBMC) and Single-Carrier-Frequency-Division Multiple Access (SC-FDMA). The proposed technique did not require a cyclic prefix and had much lower out-of-band emissions.

For video coding, Nightingale et al. [7] evaluated the performance of the HEVC regular in loss-prone networks. Zeng et al. presented a comparison between the coding gains predicted by the target models of video quality assessment for the two standards [8]. Bojkovic examined the general performance of HEVC and implementation [9].

This paper proposes a new MCS, which is called a modulation coding scheme proposed (MCS pro). The MCS pro has more throughput than the MCS standard. Then, the MCS pro is combined with FBMC. In terms of the video coding, this paper also proposes a new video coding which is called H.265 proposed ( $\mathrm{H} .265$ pro). The H.265 pro with optimum H.265 is used to find the best video coding.

\section{Overview of the LTE-Advanced}

3GPP defines three channels of control, a random-access channel (RACH), a physical channel $(\mathrm{PCH})$, and a shared data channel (SDCH), and reference signals for the LTE downlink. The control channels are transmitted in the control region at the beginning of each subframe, that is, in the 1st, 2 nd, or 3 rd orthogonal frequency-division multiplexing (OFDM) symbols [10], and the physical broadcast channel $(\mathrm{PBCH})$ is plotted on the 72 central subcarriers (6 resource blocks $(\mathrm{RB})$ ) of the available bandwidth [11]. Figure 1 illustrates how the aforementioned logical channels are mapped on physical channels in a $20 \mathrm{MHz}$ transfer scenario $(100 \mathrm{RB})[12,13]$. 3GPP describes two synchronization messages: the primary synchronization (P-SS) and secondary synchronization (S-SS), which are used for the search and synchronization procedures of the UE cells. Note that, in LTE, the time is divided into " 10 " ms frames, each frame is divided into "10" subframes, and each subframe is divided into two time slots [14]. At last, a slot is partitioned into 7 OFDM symbol images (or six slots in the situation of amplified OFDM cyclic prefix).

\section{Digital Video Coding Standard}

The core of this paper is on transcoding for bit rate saving. The different types of video coding standards H.265 and H.264 are described in the following sections.

3.1. Advanced Video Coding (AVC/H.264). The H.264 is the common coding method [15]. It aimed for a good variety of video applications from mobile phones to web applications to the TV (HDTV). A variety of the practicality enhancements inside the H.264 commonplace compared to previous codecs of video are the discrete cosine transformation works at $4 \times 4$ pixels rather than $8 \times 8$. However, it additionally supports $8 \times 8$, color sampling is compatible with $4: 2: 2$ and
$4: 4: 4$, up to twelve bits per pixel are possible. The H.264 has features such as the motion compensation (MC) blocks are of various sizes, coding is Arithmetic of Variable length (VLC), De-blocking filter and built-in reference mechanism, frequency distortion optimizer, weighted duplex forecast, redundant pictures (RD), versatile layout of the macroblocks, direct mode for frame B, multiple reference frames, and MC for subpixel [15].

\subsection{High-Efficiency Video Coding (HEVC/H.265). The H.265} procedure outlines the configurations to denote the coded data of video. Its encoder produces structures that are captured inside units of data referred to as NAL (Network Abstraction Layer) [16].

H.265 has a structure similar to H.264. H.265 has many enhancements such as flexible division partitions, transforming block sizes, and high interpolation with a complicated and unblocking filter and supports multiprocessing [16-18]. The different options concerned in hybrid video coding using H.265/HEVC are as follows:

(i) A different form of structure coding like coding tree units (CTU), coding tree block (CTB), coding units (CUs), coding blocks (CBs), prediction units (PUs) and prediction blocks (PBs) $[19,20]$

(ii) Transform units (TUs) and transform blocks (TBs)

(iii) Different MV signaling

(iv) Quantization control

(v) In-loop de-blocking filtering

(vi) Sample adaptive offset (SAO) [15]

\section{Implementation of the Proposed System in Physical Layer}

This paper proposes a new communication system as shown in Figure 2, which is called Pro-G. Figure 2 illustrates the steps of the proposed system, some of which are components of the $4 \mathrm{G}$ system with the addition of proposals including a new modulation-coding scheme (MCS) that is more efficient and replacement of OFDM with FBMC. The new system and LTE-ADV features are presented in Table 1.

This paper proposes a new modulation coding scheme (MCS pro) (Algorithm 1) and compares the proposed scheme with the traditional MCS (Table 2).

\section{Implementation of the Proposed Video Coding}

This paper also explains a new multicoding with controller transcoding video method, which is called H.265 pro. The method has been designed by using two HEVC structures, as shown in Figure 3. The figure shows the proposed multicoding step, which has same steps of traditional H.265 coding, which contains transformation (T), quantization (Q), motion composition (MC), buffer, and sample adaptive offset (SAO), but these have a multicoding with different 


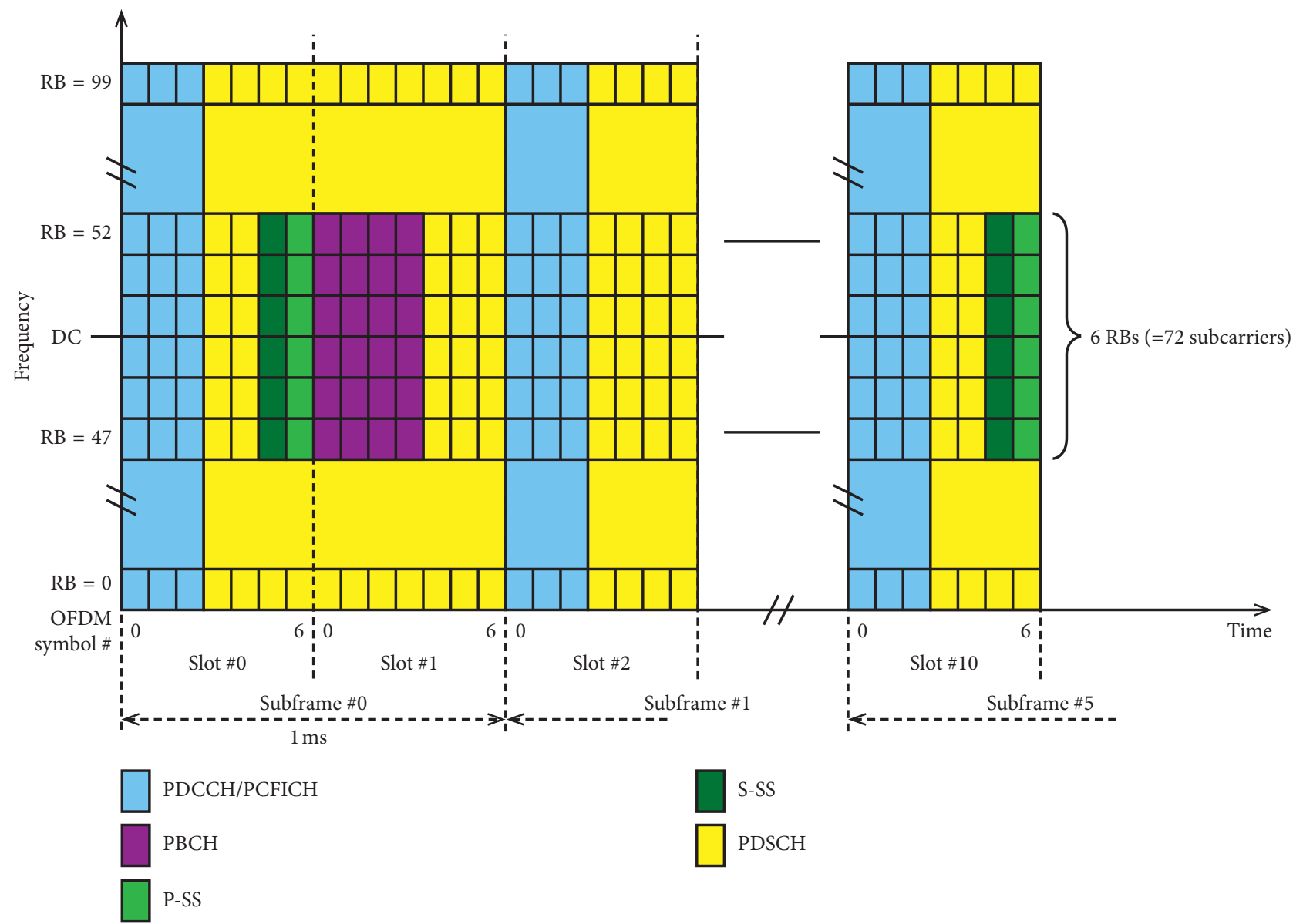

FIgURE 1: Mapping of LTE downlink channels.

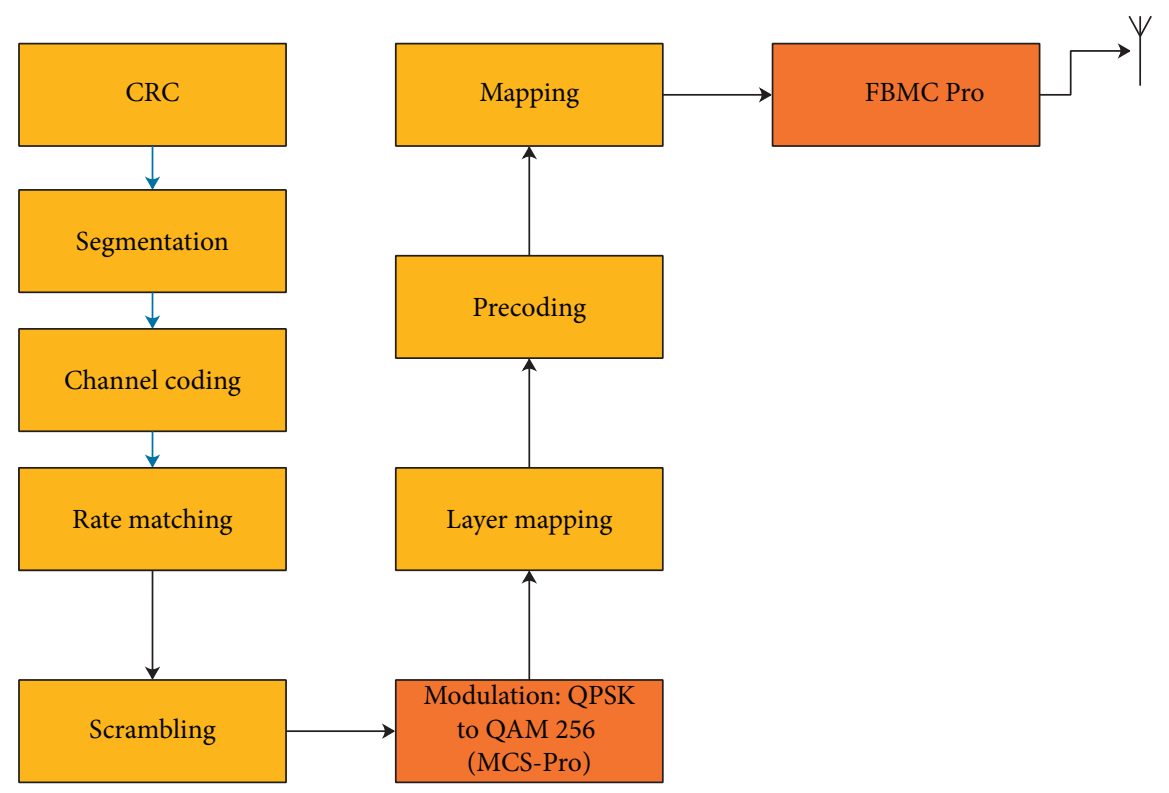

Figure 2: Block diagram of the proposed system.

parameter codings such as rate factor and dimension. First, the raw data video enters the first pass of H.265. The raw video data are analyzed and saved as a log file; then, the second pass collects the data from the first pass and achieves the best encoding quality. The second pass is controlled by bit rate ranging. 
TABLE 1: Comparison of the features of the system.

\begin{tabular}{lcc}
\hline System & LTE-ADV & Pro-G \\
\hline Bandwidth & $1.4-20 \mathrm{MHz}$ & $1.4-40 \mathrm{MHz}$ \\
Channel modulation & QPSK to QAM 256 & QPSK to QAM 256 (MCS pro) \\
Waveform modulation & OFDM & OFDM and FBMC \\
\hline
\end{tabular}

TABle 2: A parameter of the MCS pro and MCS standard.

\begin{tabular}{|c|c|c|c|c|c|c|}
\hline \multirow{2}{*}{ CQI index } & \multicolumn{3}{|c|}{ MCS standard } & \multicolumn{3}{|c|}{ MCS pro } \\
\hline & Modulation & Code rate & Efficiency & Modulation & Code rate & Efficiency \\
\hline 1 & QPSK & 78 & 0.1523 & QPSK & 449 & 0.877 \\
\hline 2 & QPSK & 193 & 0.3770 & QPSK & 602 & 1.1758 \\
\hline 3 & QPSK & 449 & 0.8770 & 16 QAM & 490 & 1.9141 \\
\hline 4 & 16 QAM & 378 & 1.4766 & 16 QAM & 553 & 2.1602 \\
\hline 5 & 16 QAM & 490 & 1.9141 & 64 QAM & 466 & 2.7305 \\
\hline 6 & 16 QAM & 616 & 2.4063 & 64 QAM & 517 & 3.0293 \\
\hline 7 & 64 QAM & 466 & 2.7305 & 64 QAM & 567 & 3.3223 \\
\hline 8 & 64 QAM & 567 & 3.3223 & 64 QAM & 616 & 3.6094 \\
\hline 9 & 64 QAM & 666 & 3.9023 & 64 QAM & 666 & 3.9023 \\
\hline 10 & 64 QAM & 772 & 4.5234 & 256 QAM & 682 & 5.332 \\
\hline 11 & 64 QAM & 873 & 5.1152 & 256 QAM & 711 & 5.5547 \\
\hline 12 & 256 QAM & 711 & 5.5547 & 256 QAM & 754 & 5.8906 \\
\hline 13 & 256 QAM & 797 & 6.2266 & 256 QAM & 797 & 6.2266 \\
\hline 14 & 256 QAM & 885 & 6.9141 & 256 QAM & 885 & 6.9141 \\
\hline 15 & 256 QAM & 948 & 7.4063 & 256 QAM & 916 & 7.1602 \\
\hline
\end{tabular}

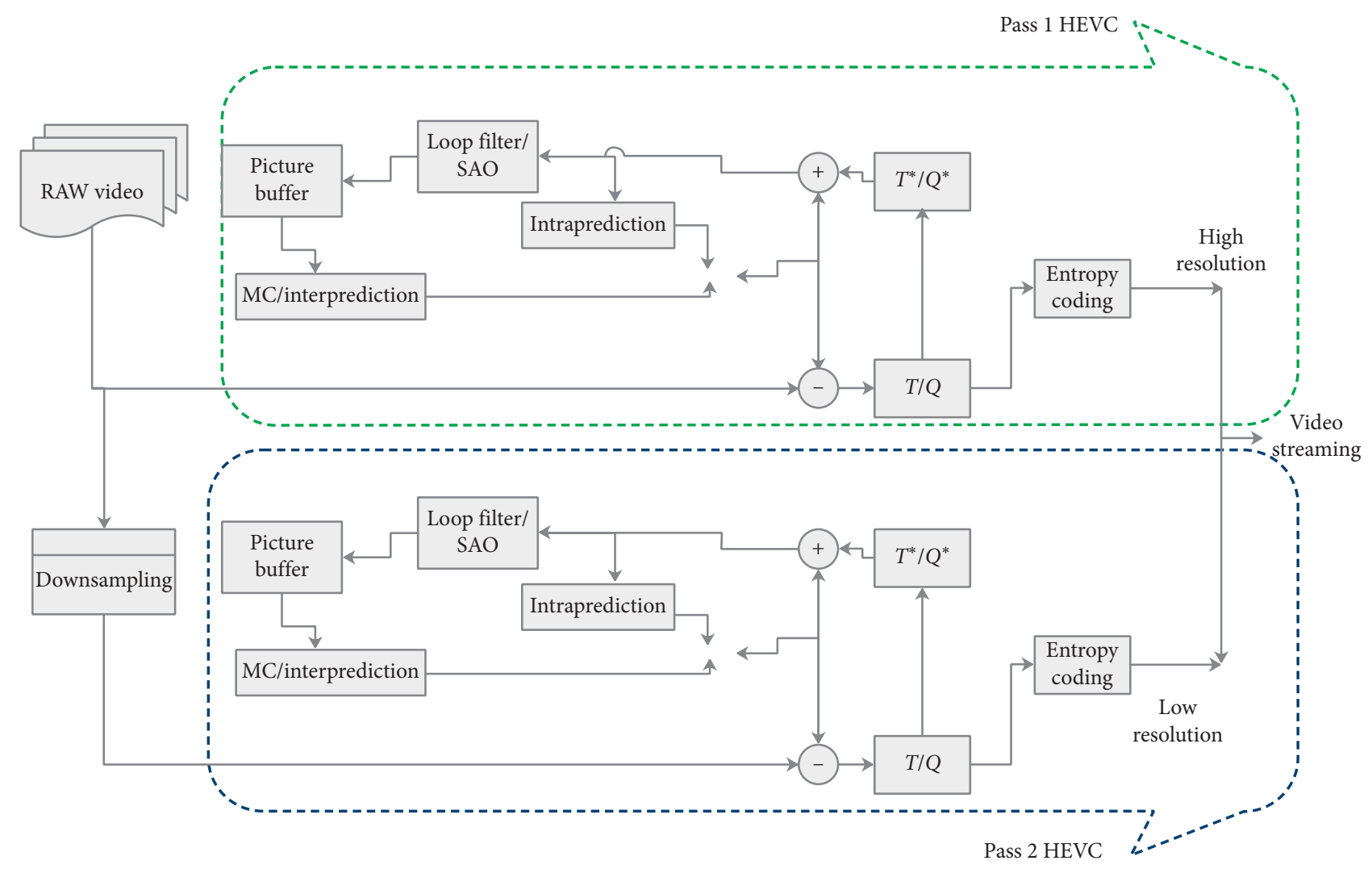

FIgURE 3: Block diagram of H.265 pro. 


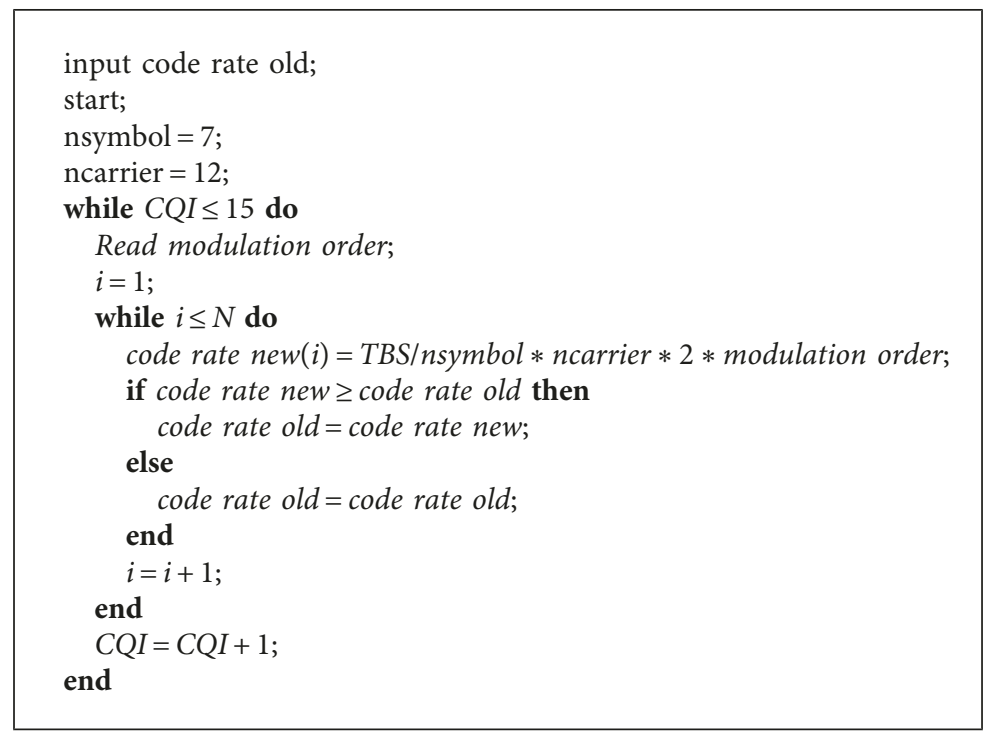

Algorithm 1: The proposed modulation coding scheme.

The H.265 pro is compared with H.265. H265 is implemented with some of the video controller methods. The methods are as follows:

(i) Constant QP: It is the amount of compression for every macroblock in a frame.

(ii) Average bit rate (ABR): the amount of data transferred per unit of time.

(iii) Video buffering verifier (VBV): It ensures that an encoded video stream can be correctly buffered.

An FFmpeg tool has been used to apply the controller types, and H.265 pro with the video sequence dimension $1920 * 1080$ and $120 \mathrm{fps}$ has been used in this paper.

\section{Implementation Results}

The simulation evaluates and compares the Pro-G and LTE$\mathrm{ADV}$ system to determine the best performance; the parameters of simulation are presented in Table 3. The MATLAB 2018 is used to implement the proposed system by using parameters in Table 3. The bandwidth is from 1.4 to $20 \mathrm{MHz}$ (i.e., $6 \mathrm{RB}$ to $100 \mathrm{RB}$ ), while the MCS is from QPSK to QAM 256; three channel types are used in the evaluation (AWGN, Flat, and Pedestrian); FBMC is used in Pro-G while OFDM in the LTE-ADV; and minimum mean squared error (MMSE) is used in the equalizer while the antenna number is from single input single output (SISO) to multiple input multiple output (MIMO). Figure 4 provides information about the simulation steps: first, it enters the number of base stations and users; second, it enters the system configuration such as the power of nodes, frequency, number of antennas, and number of RB, and the modulation type index.

Figure 5 shows the throughput of transmitted data versus SNR in the AWGN channel; the parameters used in evaluation are FBMC, SISO, and $20 \mathrm{MHz}$. The throughput in MCS pro is about $128 \mathrm{Mb} / \mathrm{s}$ while that of MCS is about $116 \mathrm{Mb} / \mathrm{s}$; the best performance is shown by the MCS pro.
TABle 3: Parameters of simulation.

\begin{tabular}{lc}
\hline Parameter & Value \\
\hline Center frequency $(\mathrm{GHz})$ & 2.5 \\
Subcarrier spacing $(\mathrm{kHz})$ & 30 \\
Bandwidth $(\mathrm{MHz})$ & $1.4-20$ \\
FFT size & 72 to 2048 \\
Number of subcarriers & 72 to 1200 \\
Channel modulation & QPSK to QAM 64 and advance \\
Waveform modulation & OFDM, FBMC \\
Channel coding & Turbo coding, $R=1 / 3$ \\
Number of antennas & $1 \times 1,2 \times 2,4 \times 4,8 \times 8$ \\
Number of frames & 100 \\
Channel type & AWGN, Flat, Ped A \\
Equalizer type & MMSE \\
Power of BS and UE $(\mathrm{dB})$ & $30 \mathrm{~dB}$ \\
\hline
\end{tabular}

Figure 6 shows the BER of the data versus SNR in the AWGN channel; the MCS pro shows the best performance because the BER in MCS pro is about $1 * 10^{-6}$ while that of MCS is about $3 * 10^{-6}$.

Figure 7 presents the throughput versus SNR between two types of modulation schemes (MCS and MCS pro) in the flat channel with different numbers of antennas.

The throughput of MCS pro is more than that of MCS; two video coding methods consumed the most throughput after $27 \mathrm{db}$. The MCS pro offers significant gains regarding the number of data rates compared to MCS. In terms of the antenna $8 \times 8$, the throughput in the MCS pro is around $965 \mathrm{Mb} / \mathrm{s}$ as opposed to around $888 \mathrm{Mb} / \mathrm{s}$ in the MCS.

Figure 8 presents the BER versus SNR between two types of modulation schemes (MCS and MCS pro) with different numbers of antenna. The BER of MCS pro is lower than that of MCS. In terms of the antenna $8 \times 8$, the BER in the MCS pro is around $3.6 * 10^{-5}$ as opposed to around $5.7 * 10^{-5}$ in the MCS.

Figure 9 illustrates the throughput versus SNR between two types of modulation schemes (MCS and MCS pro) in the 


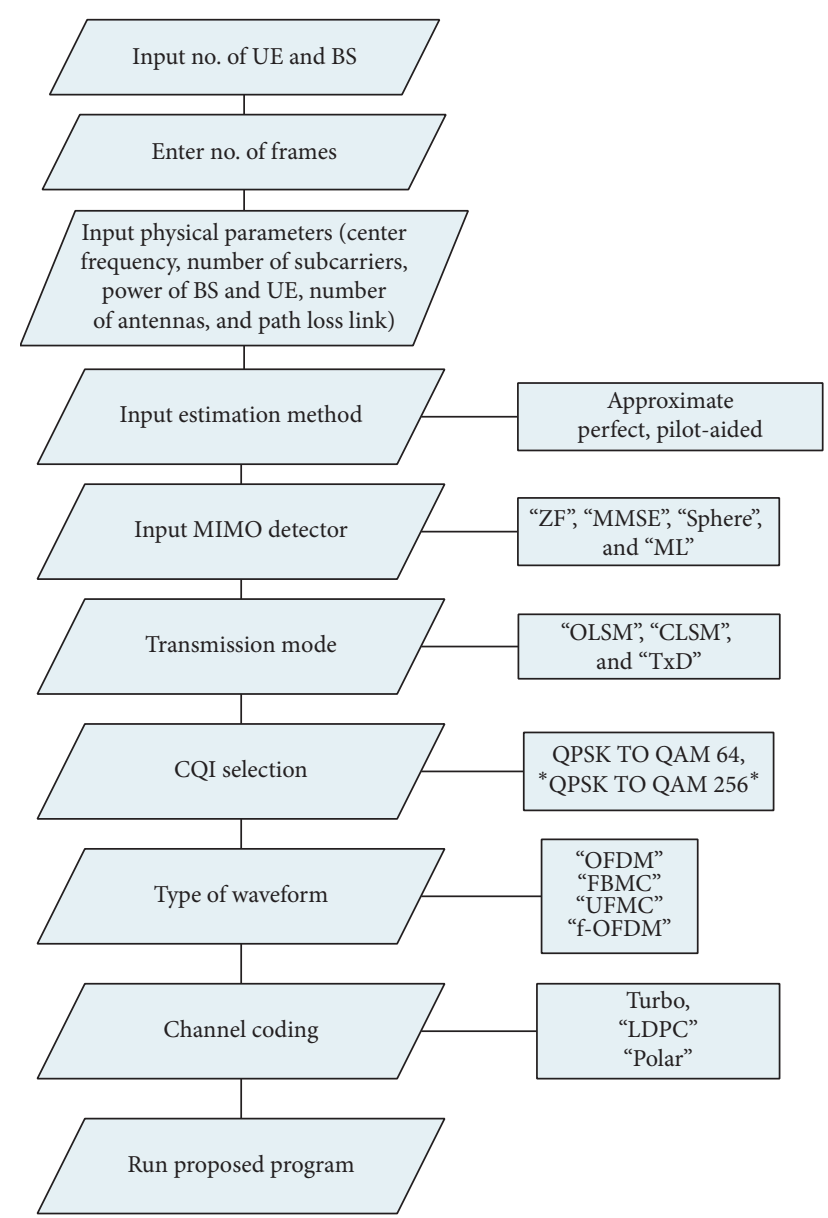

FIgURE 4: Simulation steps.

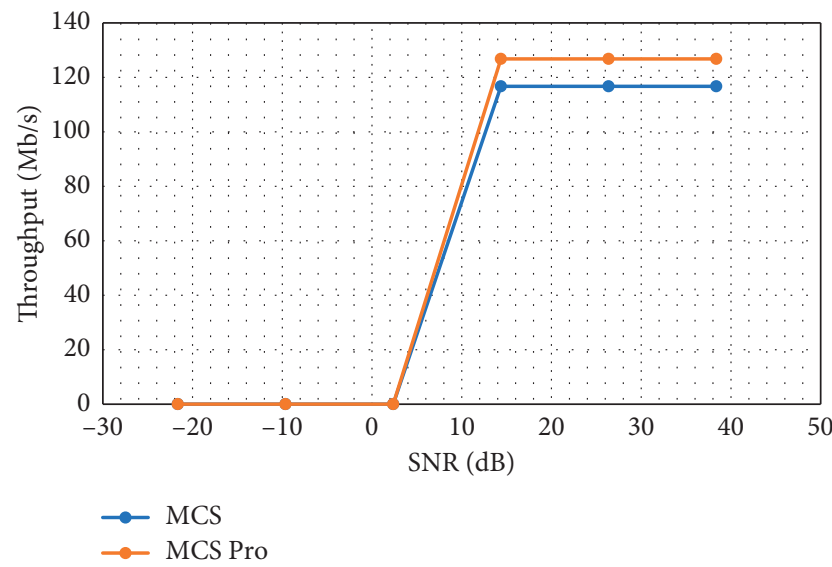

FIGURE 5: SNR versus throughput in the AWGN channel.

pedestrian channel with different numbers of antenna. The throughput is the same as that in the flat channel.

Figure 10 shows that the BER of MCS pro is lower than that of MCS. In terms of the antenna $8 \times 8$, the BER in the MCS pro is around $3 * 10^{-4}$ as opposed to around $4 * 10^{-3}$ in the MCS.

Figure 11 shows the throughput with different numbers of antenna. The parameters used in the evaluation are

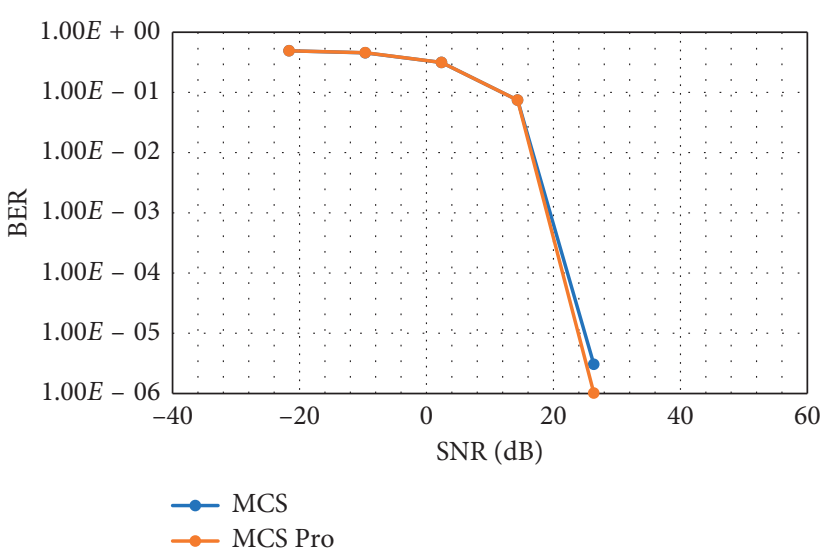

Figure 6: SNR versus BER in the AWGN channel.

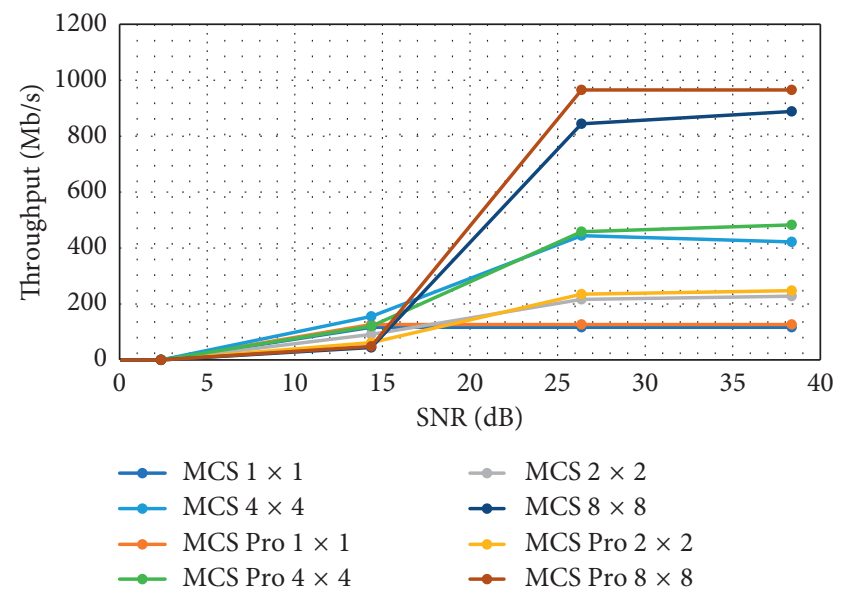

Figure 7: SNR versus throughput in the flat channel.

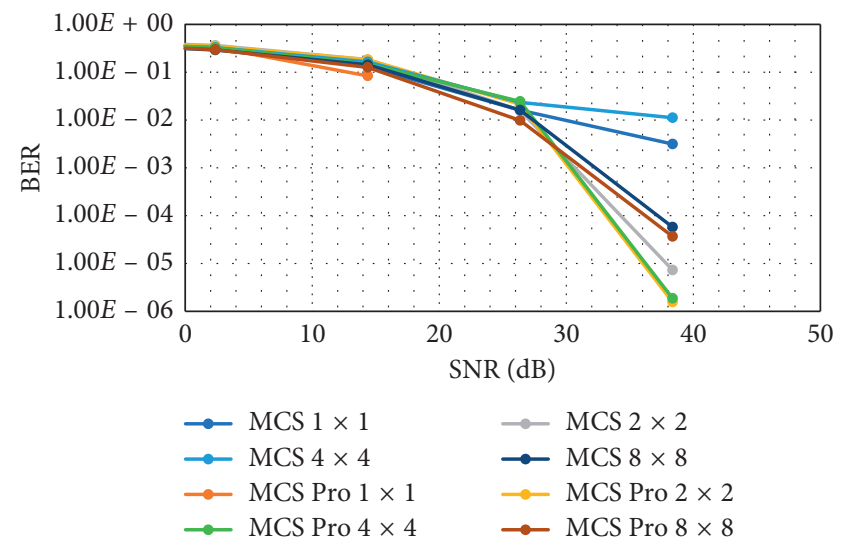

Figure 8: SNR versus BER in the flat channel.

OFDM (LTE), FBMC (Pro-G), SISO, MIMO, $20 \mathrm{MHz}$, and LTE-ADV. When the number of antennas is eight, the throughput in the Pro-G is the highest, that is, around $965 \mathrm{Mb} / \mathrm{s}$, as opposed to that of LTE-MCS pro and LTE$\mathrm{ADV}$, which was about 900 and $746 \mathrm{Mb} / \mathrm{s}$, respectively.

Figure 12 illustrates a level of bit rate with high resolution $(720,1080,4 \mathrm{~K}$, and $8 \mathrm{~K})$ between two video coding 


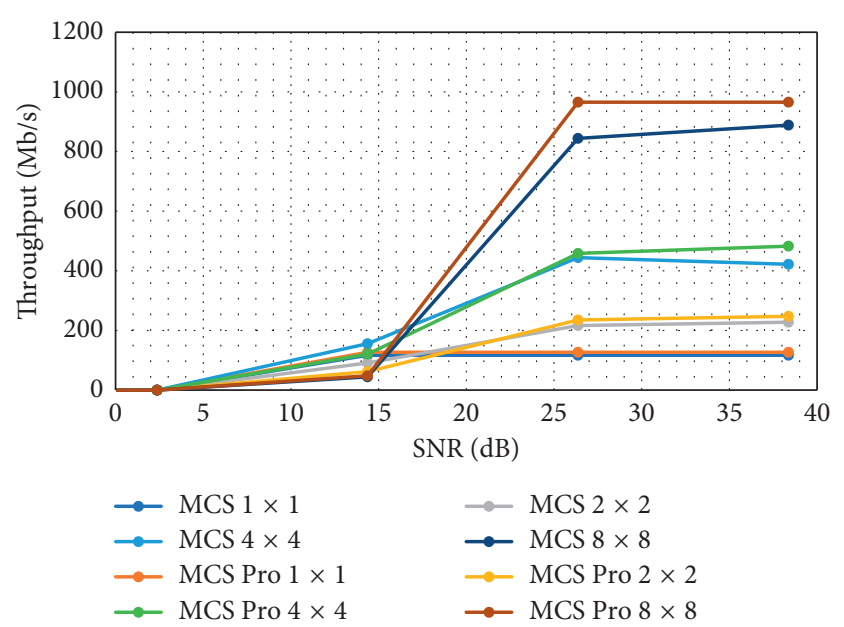

FIGURE 9: SNR versus throughput in the pedestrian channel.

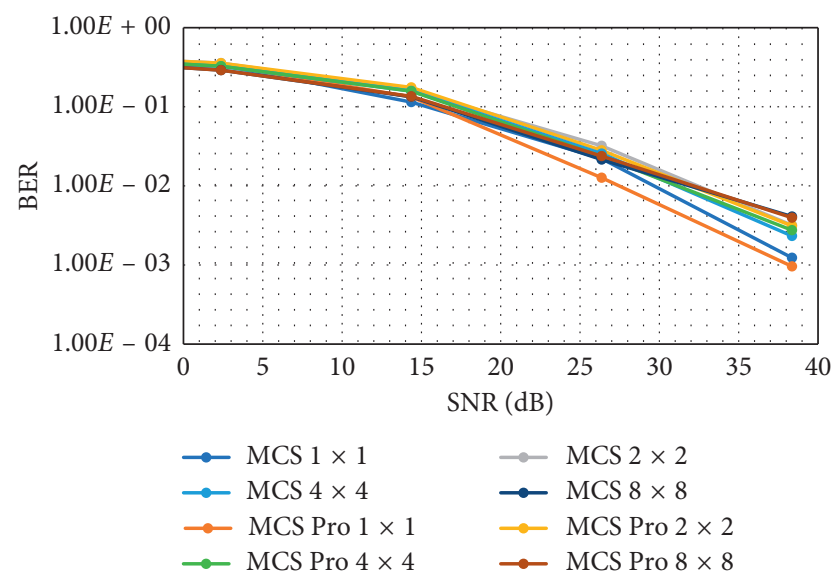

FIgURE 10: SNR versus BER in the pedestrian channel.

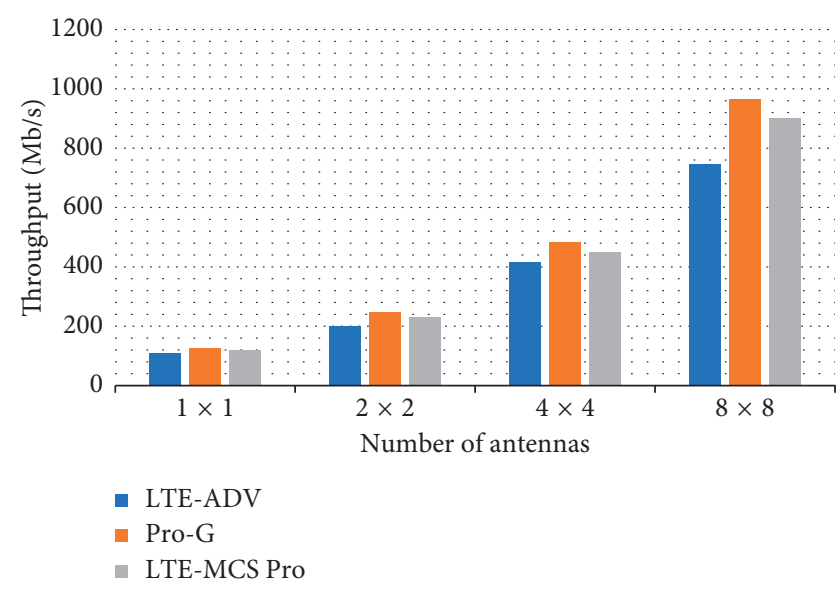

FIGURE 11: Comparison of the throughput with different numbers of antenna.

methods (H.265 and H.265 pro). The parameters used in the evaluation are shown in Table 4.

The bit rate of H.265 pro is lower than that of H.265; both video coding methods consumed most data on $8 \mathrm{~K}$. The H.265 pro offers significant gains regarding coding efficiency

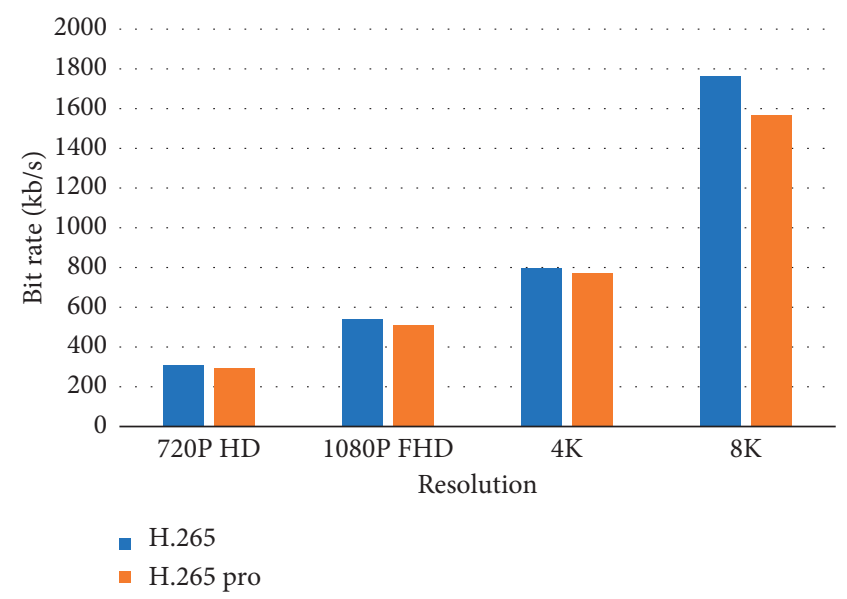

FIgURE 12: Comparison of the bit rate between the H.265 and the H.265 pro in high-resolution video.

TABle 4: Parameters of the video sequences.

\begin{tabular}{lccc}
\hline Resolution & Resolution dimension & Frame rate & Frame number \\
\hline $720 \mathrm{HD}$ & $1280 * 720$ & & \\
$1080 \mathrm{HD}$ & $1920 * 1080$ & 120 & 100 \\
$4 \mathrm{~K}$ & $3840 * 2160$ & & \\
$8 \mathrm{~K}$ & $7680 * 4320$ & & \\
\hline
\end{tabular}

compared to the H.265. When the resolution is $8 \mathrm{~K}$, the bit rate of H.265 pro in $8 \mathrm{~K}$ is lower than that of H.265 (i.e., about $1567 \mathrm{kbps}$ and $1763 \mathrm{kbps}$, respectively). Table 5 presents the evaluation parameters of the H.265 pro and H.265.

Figure 13 presents that the bit rate performance in H.265 pro is the lowest (the best performance), the resolution 1080 is used in the evaluation, and the $\mathrm{ABR}$ rate is the largest. $\mathrm{ABR}$ has a higher bit rate than H.265pro about double rate, while the bit rate in constant rate also higher than in H.265pro (about 525 and 424, respectively). The VBV is considered the best and closest to the h265 pro.

Figure 14 shows the number of users of the two video coding methods from 720 to $8 \mathrm{~K}$. The number of users was highest for H.265 pro in 720; 720 has the most number of users for both the wireless systems while the lowest number of users is in $8 \mathrm{~K}$.

In terms of 720 in the Pro-G, the H.265 pro has the highest number of users around 3286 users as opposed to that of H.265, which was about 3116 users. In LTE-ADV, the number of users was 2541 for H.265 pro compared to 2410 for H.265.

\section{Conclusions}

This paper proposes a method of wireless transmission and video encoding method where the two proposed methods are compared with the traditional method. The evaluation parameters used are the data transfer rate, the compression ratio, and the bit error rate. Both proposed systems are combined to calculate the number of users, which is compared with the traditional systems.

The results showed that the proposed method for transmitting wireless data is better than the LTE-ADV 
TABLE 5: Comparison results of the two video coding methods.

\begin{tabular}{lccc}
\hline Resolution & Parameter & H.265 pro & H.265 \\
\hline \multirow{3}{*}{$8 \mathrm{~K}$} & Data rate $(\mathrm{kb} / \mathrm{s})$ & 1567.14 & 1763.66 \\
& Time $(\mathrm{sec})$ & 75.23 & 171.46 \\
& $\mathrm{CR}$ & 30486 & 27089 \\
\hline \multirow{3}{*}{$4 \mathrm{~K}$} & Data rate $(\mathrm{kb} / \mathrm{s})$ & 772.66 & 799.63 \\
& Time $(\mathrm{sec})$ & 30.01 & 40.86 \\
& $\mathrm{CR}$ & 15458 & 14937 \\
\multirow{3}{*}{1080} & Data rate $(\mathrm{kb} / \mathrm{s})$ & 511.14 & 538.58 \\
& Time $(\mathrm{sec})$ & 10.16 & 12.47 \\
& $\mathrm{CR}$ & 5842 & 5544 \\
720 & Data rate $(\mathrm{kb} / \mathrm{s})$ & 293.75 & 309.72 \\
& Time $(\mathrm{sec})$ & 5.14 & 6.69 \\
& $\mathrm{CR}$ & 4518 & 4285 \\
\hline
\end{tabular}

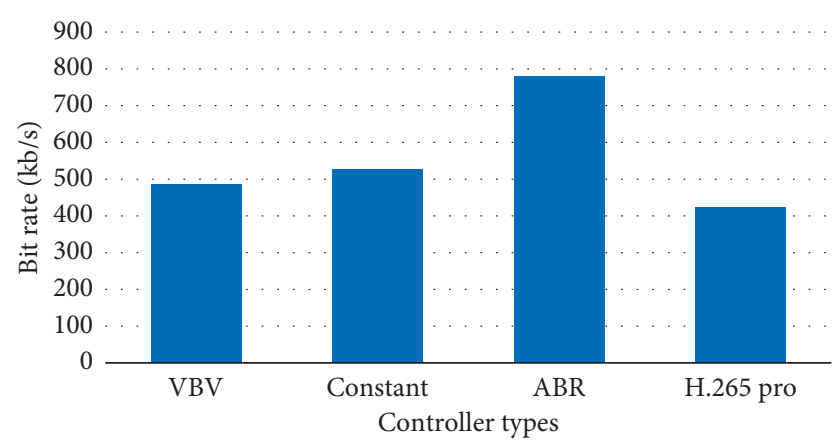

Figure 13: Comparison of bit rate video coding with the control.

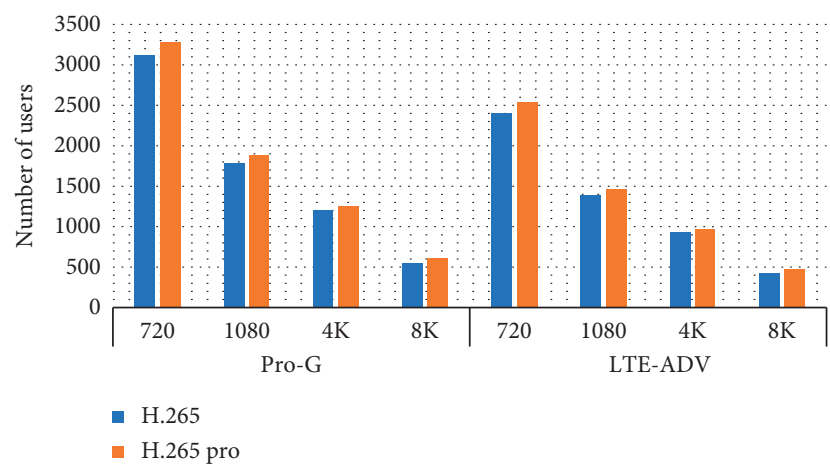

FIgURE 14: Comparison of the number of users between video coding methods and the wireless systems in a high-resolution video.

method where the throughput increased the data transfer by $29 \%$ because the proposed system uses a more efficient MCS compared to the traditional system. The proposed system uses the FBMC for waveform modulation. On the contrary, OFDM is used in the LTE-ADV. The improvement goes back to FBMC, which has less processing time and more spectral efficiency than LTE-ADV.

The bit rate has been gained in H.265 pro about $13 \%$ as compared to H.265.

\section{Data Availability}

The data used to support the findings of this study are available from the corresponding author upon request.

\section{Conflicts of Interest}

The authors declare that they have no conflicts of interest.

\section{References}

[1] P. Sule and A. Joshi, "Architectural shift from 4G to 5G wireless mobile networks," International Journal of Computer Science and Mobile Computing, vol. 3, no. 9, pp. 715-721, 2014.

[2] E. T. Tchao, J. D. Gadze, and J. O. Agyapong, "Performance evaluation of a deployed 4G LTE network," International Journal of Advanced Computer Science and Applications, vol. 9, no. 3, pp. 165-178, 2018.

[3] S. P. Erik Dahlman and J. Sköld, 4G LTE/LTE-Advanced for Mobile Broadband, Elsevier Ltd., Amsterdam, Netherlands, 2011.

[4] J. Hämälainen, The Future of LTE: The Femtocells Perspective, Aalto University, Espoo, Finland, 2013.

[5] T. Pandiaraj, "Power control optimization and improving throughput for LTE-advanced relay networks," International Journal of Innovative Technology and Exploring Engineering, vol. 3, no. 2, pp. 86-88, 2013.

[6] R. Nissel and M. Rupp, "Pruned DFT spread FBMC: low PAPR, low latency, high spectral efficiency," IEEE Transactions on Communications, vol. 66, no. 10, pp. 4811-4825, 2018.

[7] J. Nightingale, Q. Wang, and C. Grecos, "HEVStream: a framework for streaming and evaluation of high efficiency video coding (HEVC) content in loss-prone networks," IEEE Transactions on Consumer Electronics, vol. 58, no. 2, pp. 404-412, 2012.

[8] K. Zeng, A. Rehman, J. Wang, and Z. Wang, "From H.264 to HEVC: coding gain predicted by objective video quality assessment models," in Proceedings of the 2013 International Workshop on Video Processing and Quality Metrics for Consumer Electronics (VPQM2013), pp. 1-6, Scottsdale, AZ, USA, January-February 2013.

[9] Z. Milicevic and Z. Bojkovic, "HEVC vs. H.264/AVC through performance and complexity comparison," in Proceedings of the 13th International Conference on Applications of Computer Engineering ( $\left.A C E^{\prime} 14\right)$, pp. 58-61, Lisbon, Portugal, OctoberNovember 2014.

[10] R. Galih, "Carrier aggregation technique to improve capacity in LTE-advanced network," Telkomnika, vol. 14, no. 1, pp. 119-128, 2016.

[11] S. K. Ibrahim and N. N. Khamiss, "Optimization a scheduling algorithm of CA in LTE ADV," Telkomnika, vol. 16, no. 6, pp. 2514-2521, 2018.

[12] G. Soni and C. Verma, "Performance investigation of LTE systems based on NI PXIe-5644R vector signal transceiver," in Proceedings of the 2015 Global Conference on Communication Technologies (GCCT), pp. 755-760, Kanyakumari, India, April 2015.

[13] R. Zhang, Z. Zheng, M. Wang, X. Shen, and L.-L. Xie, "Equivalent capacity in carrier aggregation-based LTE-A systems: a probabilistic analysis," IEEE Transactions on Wireless Communications, vol. 13, no. 11, pp. 6444-6460, 2014.

[14] S. Mohammed Elmusrati and R. Virrankoski, "LTE-advanced: technology and performance analysis," Master's thesis, University of Vaasa, Vaasa, Finland, 2011.

[15] M. Uhrina, J. Frnda, L. Sevcik, and M. Vaculik, "Impact of H.264/AVC and H.265/HEVC compression standards on the 
video quality for $4 \mathrm{~K}$ resolution," Advances in Electrical and Electronic Engineering, vol. 12, no. 4, pp. 368-376, 2014.

[16] A. Jassal, I. S. Member, C. Leung, and I. Member, "H.265 video capacity over beyond-4G networks," in Proceedings of the 2016 IEEE International Conference on Communications (ICC), pp. 1-6, Kuala Lumpur, Malaysia, May 2016.

[17] G. J. Sullivan, J.-R. Ohm, W.-J. Han, and T. Wiegand, "Overview of the high efficiency video coding (HEVC) standard," IEEE Transactions on Circuits and Systems for Video Technology, vol. 22, no. 12, pp. 1649-1668, 2012.

[18] M. Winken, P. Helle, and D. Marpe, "Transform coding in the HEVC test model," in Proceedings of the IEEE International Conference on Image Processing, pp. 3693-3696, Taipei, Taiwan, September 2011.

[19] K. Sihag and C. S. Lamba, "Algorithm and architecture design of high efficiency video coding (HEVC) standard," International Journal of Computer Science and Mobile Computing, vol. 5, no. 10, pp. 171-178, 2016.

[20] R. Ranjana, "Video compression using compact tool (HEVC)," International Journal of Advanced Research in Computer Science and Software Engineering, vol. 6, no. 7, pp. 140-143, 2016. 


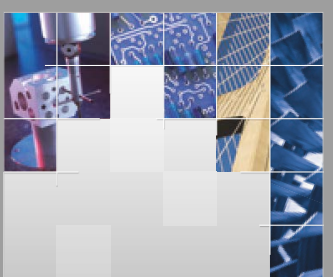

\section{Enfincering}
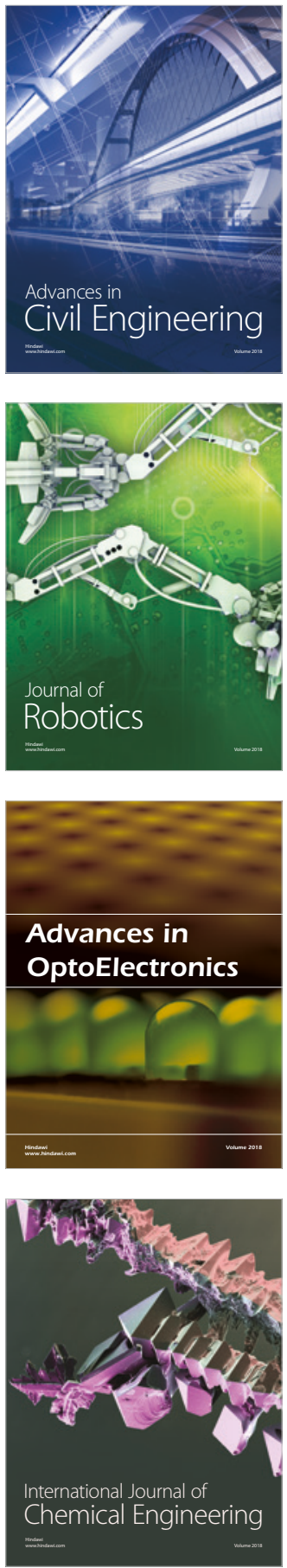

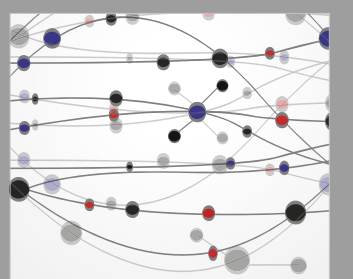

\section{Rotating \\ Machinery}

The Scientific World Journal

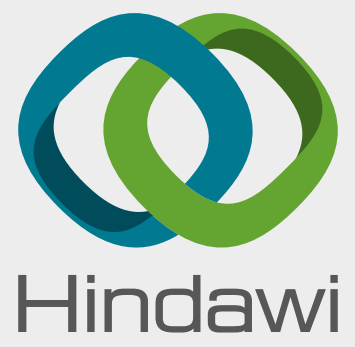

Submit your manuscripts at

www.hindawi.com
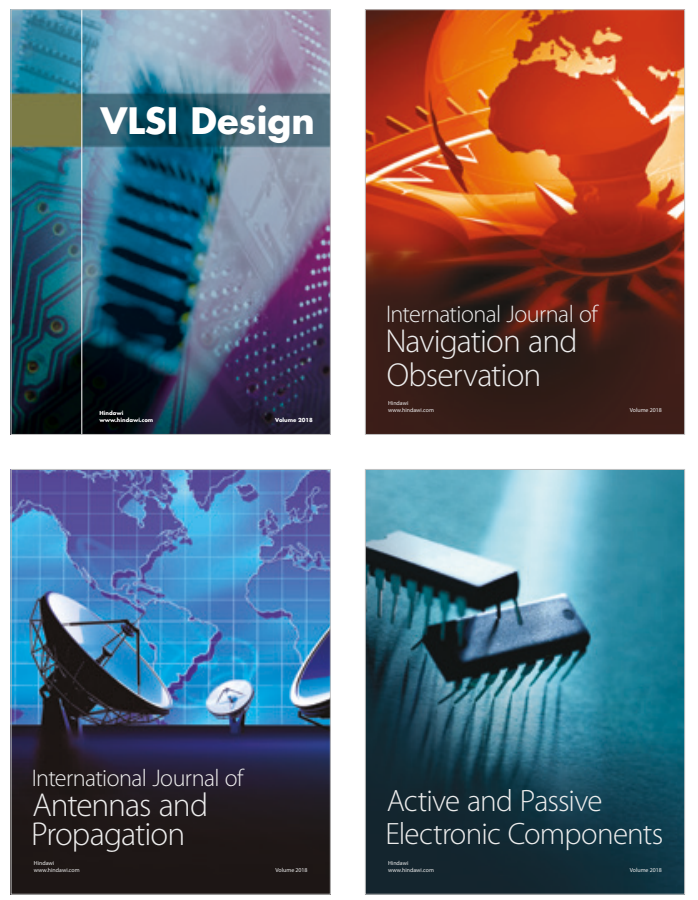
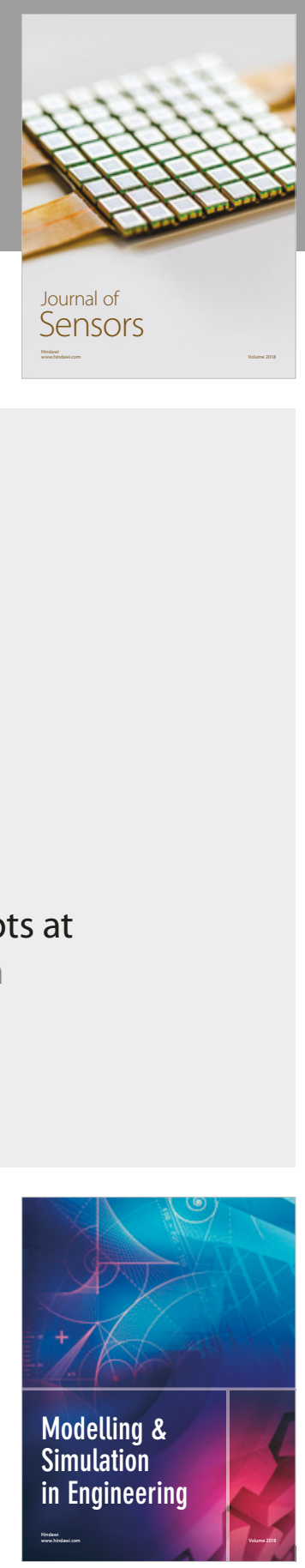

\section{Advances \\ Multimedia}
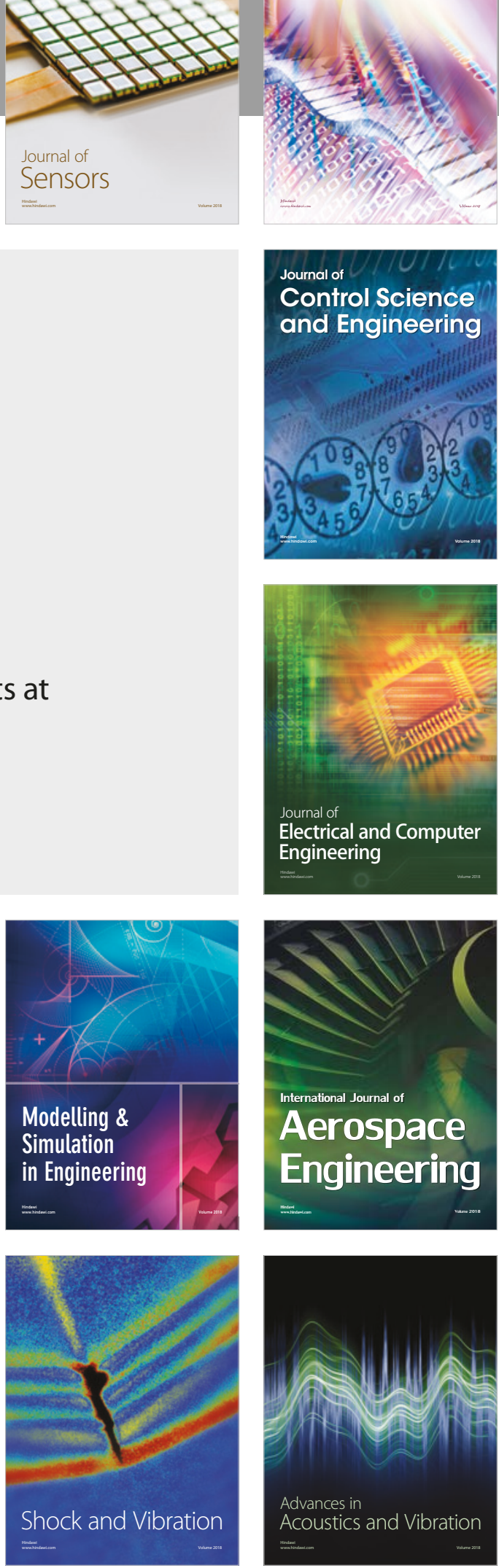